\title{
Detection and Characterization of the Evolution of Cerebral Abscesses with Diffusion-Weighted Magnetic Resonance Imaging
}

\author{
-Two Case Reports-
}

\author{
Takeshi MikAmI, Koji SAITO, Takaaki KATO, Shinsuke IRIE, \\ Junpei YOSHIKAWA, and Shiro KONDO
}

Department of Neurosurgery, Kushiro Neurosurgical Hospital, Kushiro, Hokkaido

\begin{abstract}
A 57-year-old man and a 45-year-old woman presented with cerebral abscesses. Diffusion-weighted magnetic resonance (MR) imaging and conventional MR imaging clearly showed the different stages of the course of the brain abscesses. As the abscess matured, the signal intensity of the center gradually increased to the typical high value with a low apparent diffusion coefficient (ADC) on diffusion-weighted MR imaging, and enhancement of the capsule on $T_{1}$-weighted $M R$ imaging with gadolinium. Healing of the abscess was revealed by the signal intensity of the center returning to isointense and an increase in ADC to the baseline. Surrounding edema showed an increase in ADC, followed by a return to the baseline. These changes probably reflect the pathological processes occurring in the abscesses.
\end{abstract}

Key words: cerebral abscess, diffusion-weighted magnetic resonance imaging, edema

\section{Introduction}

Diffusion-weighted magnetic resonance (MR) imaging shows cerebral abscesses as areas of increased signal intensity and markedly decreased apparent diffusion coefficient (ADC), ${ }^{3)}$ whereas all necrotic tumors or cystic portions of brain tumors appear as low signal intensity areas compared with the normal brain parenchyma. However, a case of abscess with a low signal intensity capsule and a high ADC has also been reported.6) Diffusion-weighted MR imaging of cerebral abscesses may exhibit radiological features more often associated with mature abscesses. We report the evolution of two cases of cerebral abscess using diffusion-weighted echo-planar MR imaging of the inside of the capsule and the surrounding edema, and compare the findings with those of conventional MR imaging.

\section{Case Reports}

Two patients with cerebral abscesses were examined with conventional MR imaging and diffusionweighted echo-planar MR imaging using a Signa Horizon 1.5-T unit (General Electric Medical System, Milwaukee, Wis., U.S.A.). Conventional MR imaging included axial spin-echo $\mathrm{T}_{1}$-weighted (500/24 msec [repetition time/echo time]), $\mathrm{T}_{1^{-}}$ weighted with gadolinium (Gd), $\mathrm{T}_{2}$-weighted (5000/96 msec), and fluid-attenuated inversion recovery (FLAIR) (1002/137.5 msec) sequences. Diffusion-weighted echo-planar imaging used two b values (400 and $1000 \mathrm{sec} / \mathrm{mm}^{2}$ ). Typical imaging parameters included an axial echo-planar spin-echo sequence acquired using the parameters of $6500 / 110.3 \mathrm{msec}$, 8-mm section thickness, 2-mm interscan space, a $128 \times 128$ matrix, and a $220 \times$ 220-mm field of view. Diffusion gradients were applied sequentially in three orthogonal directions to generate three sets of axial diffusion-weighted

Received July 24, 2001; Accepted November 27, 2001

Author's present address: T. Mikami, M.D., Department of Neurosurgery, Sunagawa City Medical Center, Sunagawa, Hokkaido, Japan. 
images. Average ADC maps were calculated in the $\mathrm{z}$ direction from these sets. Calculations of the trace were not performed. The ADC was measured by manually placing the square region of interest (ROI) inside the capsule and the surrounding edema, guided by FLAIR and $\mathrm{T}_{1}$-weighted imaging with $\mathrm{Gd}$. At least three different ROIs were placed within each area, with care taken to avoid partial-volume effects.

Case 1: A 57-year-old man was admitted to our hospital after a Jacksonian seizure. The initial MR sequences revealed an area of low enhancement on the $\mathrm{T}_{1}$-weighted imaging with $\mathrm{Gd}$, and an area of low signal intensity and high ADC on the diffusionweighted imaging (Figs. 1A and 2). Two days after admission, his fever spiked, and the correct diagnosis of cerebral abscess was made based on an area of high signal intensity and reduced ADC on the diffusion-weighted imaging (Fig. 2). The patient was initially treated conservatively using antibiotics. During the first 14 days, the inside of the capsule showed a gradual decrease in ADC (Fig. 2). The strongest ring-like enhancement was seen on the $\mathrm{T}_{1^{-}}$ weighted imaging with $\mathrm{Gd}$ at the time of the lowest ADC $\left(0.38 \times 10^{-3} \mathrm{~mm}^{2} / \mathrm{sec}\right)$ (Fig. 1B). The patient underwent drainage for ventricular rupture on the 28th day.

Case 2: A 45-year-old woman was admitted to our hospital after the onset of severe headache. Computed tomography and conventional MR imaging revealed a typical ring-enhanced mass that could not be differentiated from other necrotic masses. The initial diffusion-weighted echo-planar imaging showed a markedly high signal intensity (Fig. 3), with corresponding reduced ADC inside the lesion (Fig. 4). The ADC was $0.44 \times 10^{-3} \mathrm{~mm}^{2} / \mathrm{sec}$. The patient was treated conservatively using antibiotics. The cerebrospinal fluid was positive for Staphylococcus aureus.

The inside of the capsule of the abscess in both cases showed a change from high to mixed signal intensity on diffusion-weighted imaging, at the same time that the capsule of the abscess was enhanced on $T_{1}$ weighted imaging with Gd. During the healing of the abscess, diffusion-weighted imaging revealed a return to the iso signal intensity, and the ADC showed a gradual increase (Figs. 2 and 4). The region of edema showed a significant increase in ADC during the early course, and the ADC values gradually returned to the baseline during edema resolution. Both patients recovered from the neurological deterioration at the time of discharge and have been doing well.

\section{Discussion}

Diffusion-weighted MR imaging is the principal method for establishing the diagnosis of brain abscess. Diffusion-weighted MR imaging shows high signal intensity in the abscess cavity in vivo and in vitro. ${ }^{4)}$ The marked differences in signal intensity between brain abscesses and necrotic or cystic tumors on diffusion-weighted imaging may be due to the different physical and biochemical components of the central contents. Pus found in an abscess cavity is a thick, mucoid, acidic, greenish-yellow fluid that consists of inflammatory cells, bacteria, necrotic tissue, and proteinaceous exudate with high viscosity. The velocity of gross fluid movement is markedly decreased in a highly viscous fluid and restricts the microscopic diffusional motion of water molecules. $^{2,5)}$ In contrast, the cystic or necrotic cavities of brain tumors usually contain necrotic tumor tissue debris, fewer inflammatory cells than in an abscess, and more serous fluid than pus. Since the necrotic portion of tumors has a high ADC, ${ }^{7)}$ brain abscess and tumor can be distinguished by diffusionweighted MR imaging.

The present two cases demonstrate that the evolution of abscesses can be characterized accurately using diffusion-weighted and other conventional MR imaging methods. Recognition of the various diffusion-weighted MR imaging features will aid in proper treatment planning. Abscess evolution can be divided into four stages: early cerebritis, late cerebritis, early capsule formation, and late capsule formation. ${ }^{1)}$ The management and prognosis for each stage are different, so it is important to distinguish between them.

Early cerebritis is the initial phase of abscess formation. During this stage, an ill-defined, subcortical hyperintense area is seen on $\mathrm{T}_{2}$-weighted and FLAIR imaging. The initial MR sequences of Case 1 suggest this stage (Fig. 1A). $\mathrm{T}_{1}$-weighted imaging with contrast medium may disclose poorly delineated, enhanced areas within the iso- to mildly hypointense edematous region. ${ }^{8)}$ We suggest that an abscess in this stage may exhibit various intensities and ADCs, because infection at this stage is focal, and an unencapsulated mass of congested vessels is present with perivascular polymorphonuclear cell infiltrates and vasogenic edema.

During the late cerebritis stage, the infection becomes more focal as small necrotic zones coalesce. The central necrotic area is hyperintense on proton density and $\mathrm{T}_{2}$-weighted imaging. The thick, somewhat irregularly marginated rim appears as iso- to mildly hyperintense on $\mathrm{T}_{1}$-weighted imaging and relatively hypointense on $\mathrm{T}_{2}$-weighted and 

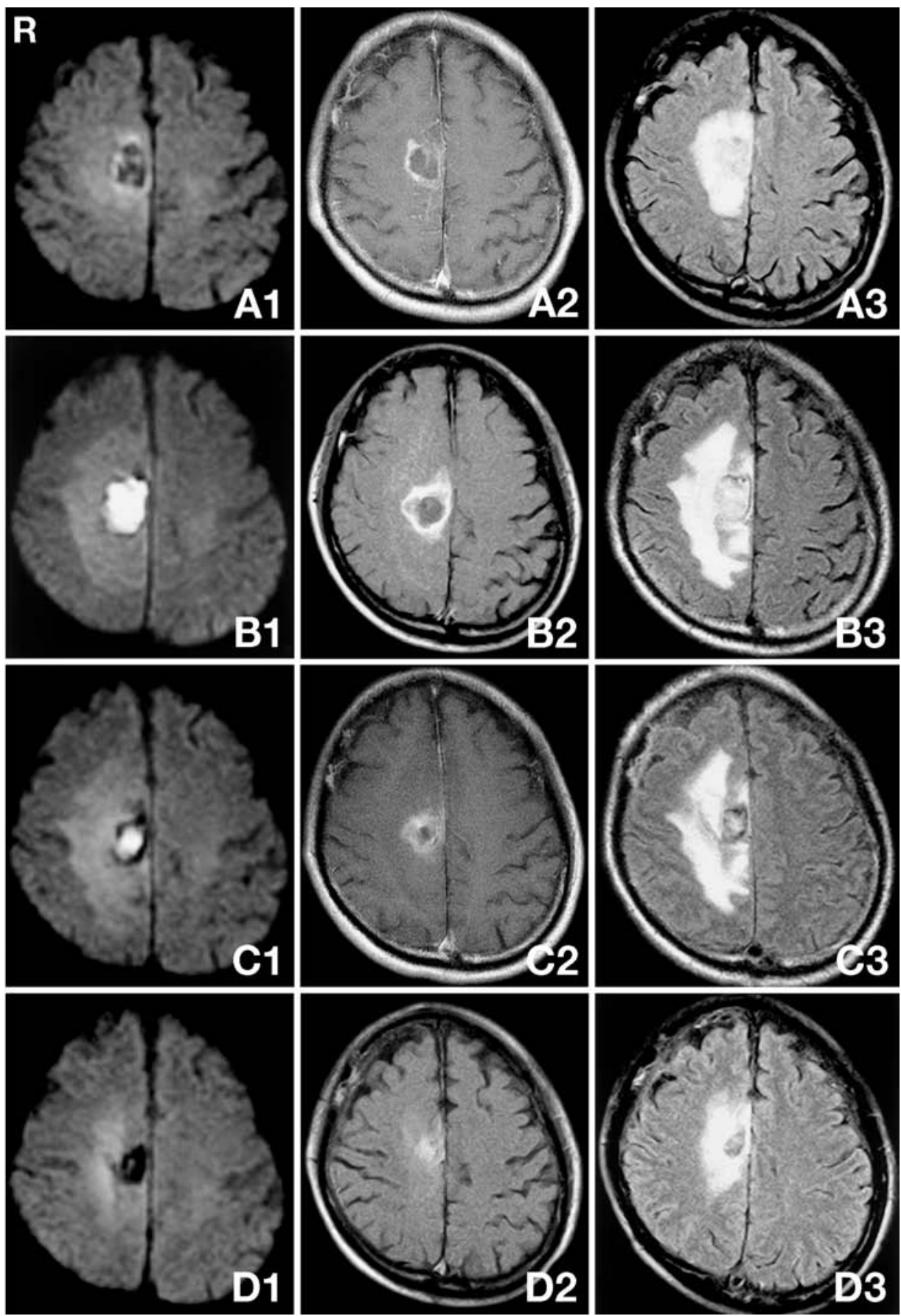

Fig. 1 Case 1. A 57-year-old man with an abscess. The initial diffusion-weighted magnetic resonance (MR) images showed a low signal intensity area (A1), and irregular and thin ring enhancement (A2). Ill-defined hyperintense area was seen on fluid-attenuated inversion recovery (FLAIR) image (A3). Fourteen days later, diffusion-weighted MR image showed a markedly high signal intensity area on the inside of the capsule (B1), and the $\mathrm{T}_{1}$-weighted MR image with gadolinium (Gd) showed thickening of the enhanced rim (B2). FLAIR image revealed peak edema at this time (B3). Thirty-three days later, diffusion-weighted MR image showed a mixed intensity area (C1), and $\mathrm{T}_{1}$-weighted $\mathrm{MR}$ image with Gd showed nodular contrast enhancement (C2). The abscess capsule became hypointense on FLAIR image (C3). Forty-one days later, the diffusion-weighted MR image showed the mixed intensity area was reduced (D1), and $T_{1}$-weighted $M R$ image with Gd showed less nodular enhancement (D2). The capsule had shrunk, and the surrounding edema was resolved on the FLAIR image (D3). 


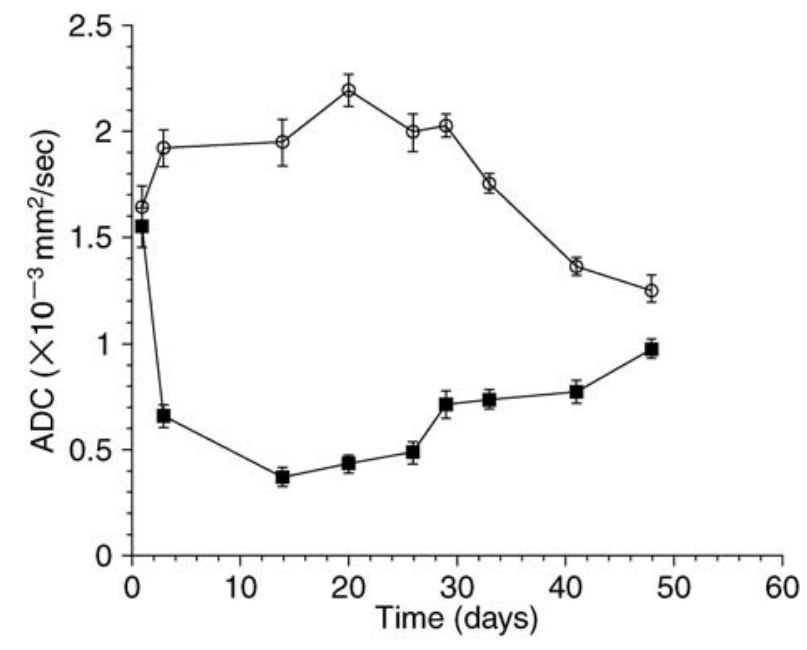

Fig. 2 Line graphs displaying change in apparent diffusion coefficient (ADC) with time in Case 1. Inside the capsule (squares), the initial ADC was increased. A significant decrease during the first 14 days was followed by a gradual increase, and the ADC returned to the baseline after 48 days. The ADC showed a significant increase in the surrounding edema (circles) during the first 20 days. This transient increase was followed by a gradual decrease and return to the baseline at 48 days.
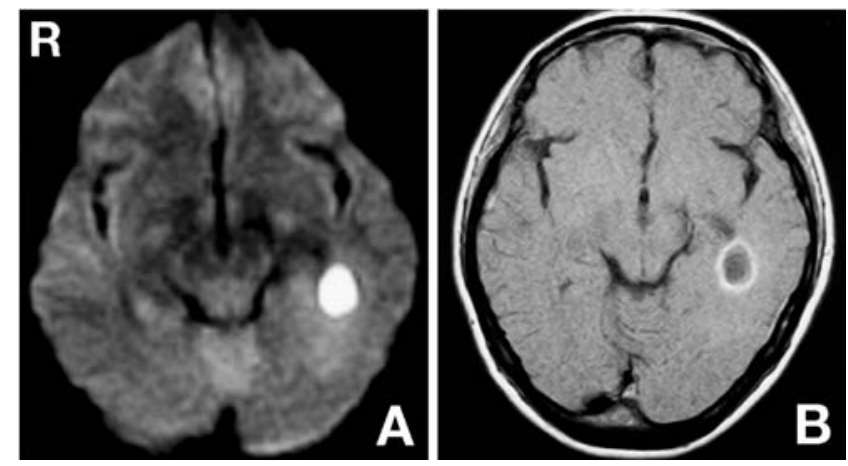

Fig. 3 Case 2. A 45-year-old woman with an abscess caused by Staphylococcus aureus infection. The initial diffusion-weighted magnetic resonance (MR) image showed a markedly high signal intensity area inside of the capsule (A), and the $T_{1}$-weighted $M R$ image with gadolinium showed the rim was diffuse and thin (B).

FLAIR imaging. $\left.{ }^{8}\right)$ Peripheral edema is nearly always present, and the typical rim enhancement is diffuse and thick. The MR sequences of Case 1 after 3 days

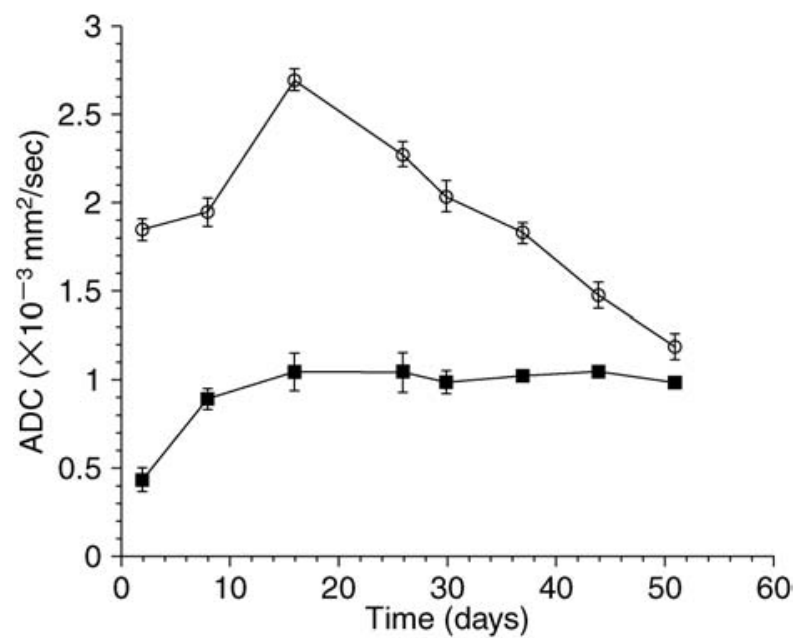

Fig. 4 Line graphs displaying the change in apparent diffusion coefficient (ADC) with time in Case 2. Inside the capsule (squares), the ADC was initially reduced, then gradually increased and returned to the baseline at $\mathbf{1 6}$ days. The ADC showed a significant increase in the surrounding edema (circles) during the first 16 days. This transient increase was followed by a gradual decrease, and the ADC returned to the baseline at 51 days.

(Fig. 1B) and the initial sequences of Case 2 (Fig. 3) suggest this stage. Diffusion-weighted MR imaging shows an abscess of this stage has a high signal intensity and a low ADC. As the central necrosis and pus mature, high cellularity and viscosity restrict the movement of water molecules.

During the early capsule stage, the collagenous abscess capsule appears as a comparatively thinwalled, well-delineated, iso- to slightly hyperintense ring that becomes hypointense on $\mathrm{T}_{2}$-weighted and FLAIR imaging. ${ }^{8)}$ The MR sequences of Case 1 (Fig. 1C2, C3) suggest this stage. Diffusion-weighted MR imaging reveals mixed intensity with hypointense rim (Fig. 1C1).

During the late capsule stage, the cavity gradually shrinks, and the surrounding edema resolves (Fig. 1D2, D3). Diffusion-weighted MR imaging reveals hypointensity (Fig. 1D1), and the ADC values gradually return to the baseline as the abscess heals. ADC is different between the cerebritis and capsule stages. ${ }^{7}$

The surrounding edema can be visualized by a substantial decrease in signal intensity and increased ADC by diffusion-weighted imaging. In our cases, the ADCs of the surrounding edema returned to the baseline, and neurological deterioration 
improved. This effect is probably due to the similar enlargement of the extracellular compartment induced by purely vasogenic edema as in peritumoral brain tissue.9) Our results suggest that diffusionweighted MR imaging is a more appropriate method to distinguish between edema types and to differentiate necrotic cells from living cells.

\section{References}

1) Britt RH, Enzmann DR: Clinical stages of human brain abscesses on serial CT scans after contrast infusion: Computerized tomographic, neuropathological, and clinical correlations. J Neurosurg 59: 972-989, 1983

2) Castillo $M$ : Imaging brain abscess with diffusionweighted and other sequences. AJNR Am J Neuroradiol 20: 1193-1194, 1999

3) Desprechins B, Stadnik T, Koerts G, Shabana W, Breucq C, Osteaux M: Use of diffusion-weighted MR imaging in differential diagnosis between intracerebral necrosis tumors and cerebral abscesses. AJNR Am J Neuroradiol 20: 1252-1257, 1999

4) Ebisu T, Tanaka C, Umeda M, Kitamura M, Naruse S, Higuchi T, Ueda S, Sato H: Discrimination of brain abscess from necrotic or cystic tumors by diffusionweighted echo planar imaging. Magn Reson Imaging
14: 1113-1116, 1996

5) Kim YJ, Chang KH, Song IC, Kim HD, Seong SO, Kim $\mathrm{YH}$, Han MH: Brain abscess and necrotic or cystic tumors: Discrimination with signal intensity on diffusion-weighted MR imaging. AJR Am J Roentgenol 171: 1487-1490, 1998

6) Krabbe K, Gideon P, Wagn P, Hansen U, Thomsen C, Madsen E: MR diffusion imaging of human intracranial tumors. Neuroradiology 39: 483-489, 1997

7) Noguchi K, Watanabe N, Nagayoshi T, Kanazawa T, Toyoshima S, Shimizu M, Seto H: Role of diffusionweighted echo-planar MRI in distinguishing between brain abscess and tumour: a preliminary report. Neuroradiology 41: 171-174, 1999

8) Osborn AG: Pyogenic parenchymal infections, in Osborn AG (ed): Diagnostic Neuroradiology. St Louis, Mosby YearBook, 1994, pp 688-692

9) Steen RG: Edema and tumor perfusion: Characterization by quantitative ${ }^{1} \mathrm{H}$ MR imaging. AJR Am J Roentgenol 158: 259-264, 1992

Address reprint requests to: T. Mikami, M.D., Department of Neurosurgery, Sunagawa City Medical Center, 1-1, West 4 North 2, Sunagawa, Hokkaido 073-0196, Japan.

e-mail: tksmikami@pop01.odn.ne.jp. 